\title{
APPROXIMATE SOLUTION OF VACCINATION, SUSCEPTIBLE, EXPOSED, INFECTED, RECOVERED (VSEIR) MODEL OF TUBERCULOSIS IN NORTH SUMATERA INDONESIA
}

\author{
Yulita Molliq Rangkuti * and Marlina Setia Sinaga \\ Department of Mathematics, Faculty of Mathematics and Natural Science, Universitas Negari Medan (UNIMED), \\ 20221, Medan, North Sumatera, Indonesia. \\ *Corresponding Author: yulitamolliq@yahoo.com
}

\begin{abstract}
In this paper, a vaccination susceptible exposed infected recovered (VSEIR) model for tuberculosis in North Sumatera is discussed. The VSEIR model is formed by a system of nonlinear differential equations. The approximate solution of this model is obtained by using the step variational iteration method (SVIM) and variational iteration method (VIM). VIM used the general Lagrange multiplier in the correction functional and runs iteratively. SVIM also used the general Lagrange multipliers for construction of the correction functional for the problems, and runs by step approach, where the computation is done by dividing the interval into subintervals with time steps. The two methods are alternative methods to obtain the approximate solutions of the VSEIR model. Comparison is made with the conventional numerical method, the fourth-order Runge-Kutta method (RK4). From the results, the SVIM solution is more accurate than the VIM solution for long time interval when compared to RK4.
\end{abstract}

(Keywords: VSEIR model, General Lagrange Multiplier, Variational Iteration Method, Step Variational Iteration Method, Fourth Order Runge Kutta)

\section{INTRODUCTION}

Tuberculosis (TB) is an airborne infection and most commonly affects the lungs. TB is a bacterial disease caused by Mycobacterium Tuberculosis, which is transmitted through contaminated air released during coughing of TB patients. TB disease can affect anyone and anywhere, and generally in children the source of infection is from adult TB patients [1]. TB infection can infect virtually the whole body because the bacteria can spread through the blood vessels or lymph nodes. Although the organs most commonly affected are the lungs, in people with low immune systems, it can infect the brain, kidneys, gastrointestinal tract, bone, lymph nodes, etc. [1].

TB is a public health problem in the world despite the efforts to control and the DOTS strategy has been implemented in many countries since 1995. In a WHO report of 2013, there were an estimated 8.6 million TB cases in 2012 where 1.1 million people $(13 \%)$ of them are in the African region. There are 450,000 people suffering TBMDR and 170,000 of them dead [2]. In North Sumatera, in 2012, around $82.67 \%$ of BTA + (infected) for 17,459 patients and around $83.34 \%$ from the total patients of TB can be cured. To see the development of transmission of Tuberculosis and the dynamics, Rangkuti et al. [5] have built a new model. The VSEIR model is divided into five classes. The class $V$ represents vaccination, $S$ represents the susceptible that do not have the disease, $E$ represents the exposed that are infected but is yet to show any sign of symptoms, $I$ represents the infective that have the disease and can transmit it to others, $R$, denotes the recovered class of those who went through infection and emerge with permanent or temporary infectionacquired immunity. The VSEIR model is described by the following dynamic system:

$$
\begin{aligned}
\dot{V}= & q N-\delta_{1} V S-\mu_{1} V, \\
\dot{S}= & \delta_{1} V S-\mu_{2} S-\alpha E S, \\
\dot{E}= & \alpha E S-\mu_{3} E-\rho E I, \\
& \dot{I}=\rho E I-\beta I, \\
& \dot{R}=\delta_{4} I-\gamma R,
\end{aligned}
$$

where $\beta=\mu_{4}+\mu_{T B}+\delta_{4}$. Here, the human birth in natural through passive vaccination $(V(t))$ at rate $p$, nonnegative parameters $\mu_{1}, \mu_{2}, \mu_{3}, \mu_{4}$ and $\gamma$ denote as natural death of population of the $V$, the $S$, the $E$, the $I$ and the $R$, respectively. Population of infected Tuberculosis died in rate $\mu_{T B}$. The susceptible population decreased due to coming individual from the $V$ in rate $\delta_{1} . \alpha$ denotes the transfer rate from susceptible to infected population. Infected population increases due to movement of individuals from infected individuals $I$ in rate $\delta_{4}$ and decreased due to movement of individuals in to the $R$ at rate $\gamma$. The model can be simplified by assuming the fractions $u=\frac{V}{N}, w=\frac{S}{N}, x=\frac{E}{N}, y=\frac{I}{N}$, and $z=\frac{R}{N}$ 
Thus, the model for TB can be simplified as follows

$$
\begin{gathered}
\frac{d u}{d t}=q-\mu_{1} u-\delta_{1} u w, \\
\frac{d w}{d t}=\left(\delta_{1} u-\mu_{2}-\alpha x\right) w, \\
\frac{d x}{d t}=\left(\alpha w-\mu_{3}-\rho y\right) x, \\
\frac{d y}{d t}=\rho x y-\beta y, \\
\frac{d z}{d t}=\delta_{4} y-\gamma z,
\end{gathered}
$$

subject to initial conditions

$u(0)=0.02167 ; w(0)=0.8174 ; x(0)=$ $0.1583 ; y(0)=0.0017$ and $z(0)=0.00092$.

The above initial conditions were obtained from the real data for TB in North Sumatera [8].

Most model of real life problems, however, are still very difficult to solve. Our motivation for this work is to provide an alternative analytical method to find the solution for the epidemic model. This is centralized on a newly modified version of VIM, which is generally called the step variational iteration method (SVIM) proposed by Yulita Molliq et al. [3]. We will present comparative solutions from VIM and fourth-order Runge-Kutta method (RK4). We choose the conventional RK4 as our benchmark, as it is widely accepted and exactly used. The accuracy of SVIM has been shown for solving two chaotic systems i.e. Rössler and Genesio systems. In SVIM technique, each interval on VIM is divided into subintervals with time span $t$ and the solution at each subinterval will be obtained. It is necessary to satisfy the initial conditions at each subinterval, where the initial conditions will be changed for each subinterval. Yulita Molliq et al. [4] modified the SVIM to find the approximate solution of a fractional biochemical reaction model.

This paper is organized as follows: Section 2 discusses the main idea on VIM and some recent advances on the technique, Section 3 deals with the concept of SVIM, Section 4 outlines the application of SVIM to the VSEIR model, Section 5 present the results obtained by the methods mentioning with some critical discussions, and lastly, Section 6 offers some concluding remarks on the method used.

\section{VARIATIONAL ITERATION METHOD (VIM)}

To introduce the basic concepts of variational iteration method (VIM), we consider the following nonlinear differential equation:

$$
L u_{i}(t)+N u_{i}(t)=g_{i}(t)
$$

where $L$ is a linear operator, $N$ is a nonlinear operator, and $g_{i}(t)$ is an inhomogeneous term $i=1,2, \cdots, n$.
According to VIM, one can construct a correction functional as follows:

$$
u_{i, k+1}=u_{i, k}+\int_{0}^{t} \lambda_{i}\left(L u_{i}+N u_{i}-g_{i}\right) d \xi
$$

where $\lambda_{i}, i=1,2,3, \ldots, n$ are the Lagrange multipliers [6], which can be identified optimally via the variational theory, $\tilde{u}_{i, n}(\xi)$ and are considered as restricted variations, i.e. $\widetilde{\delta u}_{i, n}(\xi)=0$. Once we have determined the Lagrange multiplier, we use VIM to perform the iteration using the initial approximation, which we choose by a linearized solution of the equation that satisfies the initial conditions. Therefore, we can successively approximate or even reach the exact solution by using

$$
u(t)=\lim _{n \rightarrow \infty} u_{i, n}(t)
$$

where $n$ is the iteration step.

\section{STEP VARIATIONAL ITERATION METHOD (SVIM)}

In this section, we shall now look at how this new modification of VIM so called step variational iteration method (SVIM) to find the approximate solution for longer time span $t$, Here, interval $[0, T]$ is regarded as an interval, then the interval is divided to subinterval with time span $t$ and the solution at each subinterval of Eqs. (7)-(10) will be obtained. It is necessary to satisfy the initial condition at each subinterval, the initial conditions $u_{1,0}, u_{2,0}, \ldots, u_{m, 0}$ will be changed for each subinterval, i.e. and it should be satisfied through initial conditions, $u_{i, n}\left(t^{*}\right)=0$ for all $n \geq 1$. Thus the formula can be written as [3]:

$$
u_{i, k+1}=u_{i, k}+\int_{0}^{t-t^{*}} \lambda_{i}\left(L u_{i}+N u_{i}-g_{i}\right) d \xi
$$

Here, $t-t^{*}$ as upper limit of integration instead of fixed upper limit of $t$ in Eq. (12). The approximate solution takes the form:

$$
u_{i}(t) \approx u_{i, n}\left(t-t^{*}\right)
$$

Where $t^{*}$ start from $t_{0}=0$ until $t_{j}=T, j$ is number of subinterval. To carry out the solution on every subinterval of equal length $\Delta t$ the values of the following initial condition are shown below

$$
c_{i}^{*}=u_{i}\left(t^{*}\right), i=1,2, \ldots, m .
$$

In general, we do not have the information of our clearance except at the initial point $t=t_{0}=0$, but these values can be obtained by assuming that the new initial condition is the solution in previous interval i.e. if the solution in interval $\left[t_{j}, t_{j+1}\right]$ is necessary then the initial condition of this interval will be as follows

$$
c_{i}=u_{i} \approx u_{i, n}\left(t_{j}, t_{j-1}\right),
$$

where $c_{i}, i=1,2, \ldots m$ are the initial conditions in the interval $\left[t_{j}, t_{j+1}\right]$. 


\section{APPLICATION}

In this section, the VIM method is applied to compute an approximate solution of nonlinear system of differential equations describing a VSEIR model [5]. According to VIM the correctional functional constructing as follow

$$
\begin{gathered}
u_{k+1}=u_{k}+\int_{0}^{t} \lambda_{1}\left(\frac{d u_{k}}{d \xi}-q+\mu_{1} u_{k}\right. \\
\left.+\delta_{1} u_{k} w_{k}\right) d \xi \\
w_{k+1}=w_{k}+\int_{0}^{t} \lambda_{2}\left(\frac{d w_{k}}{d \xi}\right) \\
-\left(\left(\delta_{1} u_{k}-\mu_{2}\right.\right. \\
\left.\left.\left.-\alpha x_{k}\right) w_{k}\right)\right) d \xi \\
x_{k+1}=x_{k}+\int_{0}^{t} \lambda_{3}\left(\frac{d x_{k}}{d \xi}-\left(\left(\alpha w_{k}-\mu_{3}\right.\right.\right. \\
\left.\left.\left.-\rho y_{k}\right) x_{k}\right)\right) d \xi \\
y_{k+1}=y_{k}+\int_{0}^{t} \lambda_{4}\left(\frac{d y_{k}}{d \xi}-\left(\rho x_{k} y_{k}-\beta y_{k}\right)\right) d \xi, \\
z_{k+1}=z_{k}+\int_{0}^{t} \lambda_{5}\left(\frac{d z_{k}}{d \xi}-\left(\delta_{4} y_{k}-\gamma z_{k}\right)\right) d \xi,
\end{gathered}
$$

where $\lambda_{i}, i=1,2,3, \lambda_{i}$ are the general Lagrange multiplier which can be identified optimally via the variational theory and the subscript $k$ indicates the $n$ th. To obtain the optimal $\lambda_{i}(\xi)$, we proceed as follows:

$$
\begin{aligned}
\delta u_{k+1}=\delta u_{k}+\int_{0}^{t} & \delta \lambda_{1}\left(\frac{d u_{k}}{d \xi}-q+\mu_{1} u_{k}\right. \\
+ & \left.\delta_{1} \widetilde{u_{k} w_{k}}\right) d \xi \\
\delta w_{k+1}=\delta w_{k}+ & \int_{0}^{t} \delta \lambda_{2}\left(\frac{d w_{k}}{d \xi}\right. \\
& -\left(\delta_{1} \widetilde{u_{k} w_{k}}-\mu_{2} w_{k}\right. \\
& \left.\left.-\alpha \widetilde{x_{k} w_{k}}\right)\right) d \xi \\
\delta x_{k+1}=\delta x_{k}+ & \int_{0}^{t} \delta \lambda_{3}\left(\frac{d x_{k}}{d \xi}\right. \\
& -\left(\alpha \widetilde{w_{k} x_{k}}-\mu_{3} x_{k}\right. \\
& \left.\left.-\rho \widetilde{y_{k} x_{k}}\right)\right) d \xi
\end{aligned}
$$

$$
\begin{array}{r}
\delta y_{k+1}=\delta y_{k}+\int_{0}^{t} \delta \lambda_{4}\left(\frac{d y_{k}}{d \xi}\right. \\
\left.-\left(\rho \widetilde{x_{k} y_{k}}-\beta y_{k}\right)\right) d \xi, \\
\delta z_{k+1}=\delta z_{k}+\int_{0}^{t} \delta \lambda_{5}\left(\frac{d z_{k}}{d \xi}\right. \\
\left.-\left(\delta_{4} \tilde{y}_{k}-\gamma z_{k}\right)\right) d \xi,
\end{array}
$$

where $\widetilde{u_{k} w_{k}}, \widetilde{x_{k} y_{k}}, \widetilde{x_{k} w_{k}}$ and $\widetilde{y_{k}}$ are considered restricted variations i.e. $\delta u_{k} \widetilde{w_{k}}=0, \delta x_{k} \widetilde{y_{k}}=0$, $\delta x_{k} \widetilde{w_{k}}=0$ and $\widetilde{\delta y_{k}}=0$, Then we have

$$
\begin{gathered}
\delta u_{k+1}=\delta u_{k}+\int_{0}^{t}\left(\delta \lambda_{1} \frac{d u_{k}}{d \xi}+\delta \lambda_{1} \mu_{1} u_{k}\right) d \xi \\
\delta w_{k+1}=\delta w_{k}+\int_{0}^{t}\left(\delta \lambda_{2} \frac{d w_{k}}{d \xi}+\delta \lambda_{2} \mu_{2} w_{k}\right) d \xi \\
\delta x_{k+1}=\delta x_{k}+\int_{0}^{t}\left(\delta \lambda_{3} \frac{d x_{k}}{d \xi}+\delta \lambda_{3} \mu_{3} x_{k}\right) d \xi \\
\delta y_{k+1}=\delta y_{k}+\int_{0}^{t}\left(\delta \lambda_{4} \frac{d y_{k}}{d \xi}+\delta \lambda_{4} \beta y_{k}\right) d \xi \\
\delta z_{k+1}=\delta z_{k}+\int_{0}^{t}\left(\delta \lambda_{5} \frac{d z_{k}}{d \xi}+\delta \lambda_{5} \gamma z_{k}\right) d \xi
\end{gathered}
$$

Thus, the general Lagrange multiplier are obtained as follow

$$
\begin{aligned}
\lambda_{1} & =-e^{-\mu_{1}(\xi-\mathrm{t})} \\
\lambda_{2} & =-e^{-\mu_{2}(\xi-\mathrm{t})} \\
\lambda_{3} & =-e^{-\mu_{3}(\xi-\mathrm{t})} \\
\lambda_{4} & =-e^{-\beta(\xi-\mathrm{t})} \\
\lambda_{5} & =-e^{-\gamma(\xi-\mathrm{t})}
\end{aligned}
$$

Here, the general Lagrange multiplier in (34)-(37) is expanded by Taylor series only one term, so the general Lagrange multiplier can be written as follows

$$
\begin{aligned}
& \lambda_{1}=-1 \\
& \lambda_{2}=-1 \\
& \lambda_{3}=-1 \\
& \lambda_{4}=-1 \\
& \lambda_{5}=-1
\end{aligned}
$$

Substitute the general Lagrange multiplier into (38)(42) into the correctional iteration functional in Eqs. (19)-(22) result in the following iteration formula:

$$
\begin{aligned}
u_{k+1}=u_{k}-\int_{0}^{t} & \left(\frac{d u_{k}}{d \xi}-q+\mu_{1} u_{k}\right. \\
& \left.+\delta_{1} u_{k} w_{k}\right) d \xi, \\
w_{k+1}=w_{k}-\int_{0}^{t} & \left(\frac{d w_{k}}{d \xi}\right. \\
& -\left(\left(\delta_{1} u_{k}-\mu_{2}\right.\right. \\
& \left.\left.\left.-\alpha x_{k}\right) w_{k}\right)\right) d \xi,
\end{aligned}
$$




$$
\begin{gathered}
x_{k+1}=x_{k}-\int_{0}^{t}\left(\frac{d x_{k}}{d \xi}\right. \\
-\left(\left(\alpha w_{k}-\mu_{3}\right.\right. \\
\left.\left.\left.-\rho y_{k}\right) x_{k}\right)\right) d \xi, \\
y_{k+1}=y_{k}-\int_{0}^{t}\left(\frac{d y_{k}}{d \xi}-\left(\rho x_{k} y_{k}-\beta y_{k}\right)\right) d \xi, \\
z_{k+1}=z_{k}-\int_{0}^{t}\left(\frac{d z_{k}}{d \xi}-\left(\delta_{4} y_{k}-\gamma z_{k}\right)\right) d \xi,
\end{gathered}
$$

The interval $[0, T]$ is divided to subintervals with the time step $\Delta t$ to obtain the solution at each subinterval. In this case, the initial conditions is satisfied at each of the subinterval [7], i.e $u\left(t^{*}\right)=c_{1}^{*}=$ $u_{0}, w\left(t^{*}\right)=c_{2}^{*}=w_{0}, x\left(t^{*}\right)=c_{3}^{*}=x_{0}$, $y\left(t^{*}\right)=c_{4}^{*}=y_{0}$ and $z\left(t^{*}\right)=c_{5}^{*}=z_{0}$. The initial conditions should be satisfied $u_{k}\left(t^{*}\right)=$ $0, w_{k}\left(t^{*}\right)=0, x_{k}\left(t^{*}\right)=0, y_{k}\left(t^{*}\right)=0$ and $z_{k}\left(t^{*}\right)=0$ for all $n \geq 1$. Such that (7) to (10) can be written as

$$
\begin{aligned}
u_{k+1}=u_{k}-\int_{0}^{t-t^{*}} & \left(\frac{d u_{k}}{d \xi}-q+\mu_{1} u_{k}\right. \\
& \left.+\delta_{1} u_{k} w_{k}\right) d \xi \\
w_{k+1}=w_{k}-\int_{0}^{t-t^{*}} & \left(\frac{d w_{k}}{d \xi}\right. \\
& -\left(\left(\delta_{1} u_{k}-\mu_{2}\right.\right. \\
& \left.\left.\left.-\alpha x_{k}\right) w_{k}\right)\right) d \xi
\end{aligned}
$$

$$
\begin{gathered}
x_{k+1}=x_{k}-\int_{0}^{t-t^{*}}\left(\frac{d x_{k}}{d \xi}\right) \\
-\left(\left(\alpha w_{k}-\mu_{3}\right.\right. \\
\left.\left.\left.-\rho y_{k}\right) x_{k}\right)\right) d \xi \\
y_{k+1}=y_{k}-\int_{0}^{t-t^{*}}\left(\frac{d y_{k}}{d \xi}-\left(\rho x_{k} y_{k}-\beta y_{k}\right)\right) d \xi, \\
z_{k+1}=z_{k}-\int_{0}^{t-t^{*}}\left(\frac{d z_{k}}{d \xi}-\left(\delta_{4} y_{k}-\gamma z_{k}\right)\right) d \xi,
\end{gathered}
$$

\section{RESULTS AND DISCUSSION}

The Maple mathematical software was used for all our computations. The iterative schemes for VIM in (44)(47) and SVIM in (49)-(52) are coded in the computer algebra package Maple and we employed Maple's built in fourth-order Runge-Kutta procedure rk4. We revised the parameters and initial conditions in [5] due to the updating of data for TB in North Sumatera in which the parameters are determined from previous studies and the Health Department of North Sumatera province as shown in Table 1.

Table 1. Parameter Values

\begin{tabular}{llc}
\hline Name of Parameter & Values & Reference \\
\hline birth rate $(q)$ & 0.0094 & {$[8]$} \\
death rate for baby $\left(\mu_{1}\right)$ & 0.0065 & {$[8]$} \\
rate of baby vaccine convert to susceptible $\left(\delta_{1}\right)$ & 0.0160 & {$[8]$} \\
death rate $\left(\mu_{2}\right)$ & 0.0075 & {$[8]$} \\
rate of susceptible to exposed $(\alpha)$ & 0.0016 & {$[8]$} \\
death rate $\left(\mu_{3}\right)$ & 0.0009 & {$[9]$} \\
rate of exposed to infected $(\rho)$ & 0.00859 & {$[8]$} \\
death rate cause TB $\left(\mu_{T B}\right)$ & 0.0071 & {$[8]$} \\
death rate $\left(\mu_{4}\right)$ & 0.0009 & {$[9]$} \\
rate of infected to recovery $\left(\delta_{4}\right)$ & 0.0919 & {$[8]$} \\
death rate in recovery period $(\gamma)$ & 0.0009 & {$[9]$} \\
\hline
\end{tabular}

Here, the number of population $(N)$ is $13,215,401$ including all babies born i.e. 303,327 babies, number of vaccination is 284,633 . The number of susceptible is $10,802,233$, exposed is 2114464 , infected is 22,360 , recovery is 12154, and mortality due to $\mathrm{TB}$ is 117 . Thus the initial conditions used are $u(0)=$ $0.02167, w(0)=0.8174, x(0)=0.1583$, $y(0)=0.0017$ and $z(0)=0.00092$ for all computations. We determine the accuracy of RK4 for the solution of Model in (7)-(10) shown in Figure 1, since the RK4 is widely used. This solution is viewed as a benchmark for this model. We used 4 iterate VIM and SVIM to find the spreading number of vaccination $(u), \quad$ susceptible $(w), \quad$ exposed $(x)$, 
infected $(y)$, and recovery $(z)$ probability at time step $\Delta t=0.0001$, respectively. The comparisons displayed between results from VIM, SVIM and RK4 for $t \in[0,60]$, in figure 2 . From the figure, it is obvious that VIM exhibits unpredictable behaviour because the graph diverts from the RK4 and SVIM. Both RK4 and SVIM solutions show good

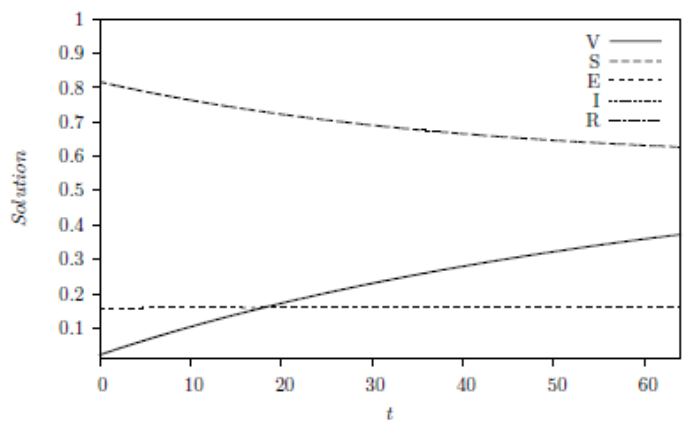

(a) synchronization at the time carried out and both the results agree very well with each other. We note that the solution of all variables will converge to RK4 solution in certain time. Table 2 presents the absolute value of $4^{\text {th }}$ iterate SVIM and VIM for recovery $\operatorname{case}(z(t))$.

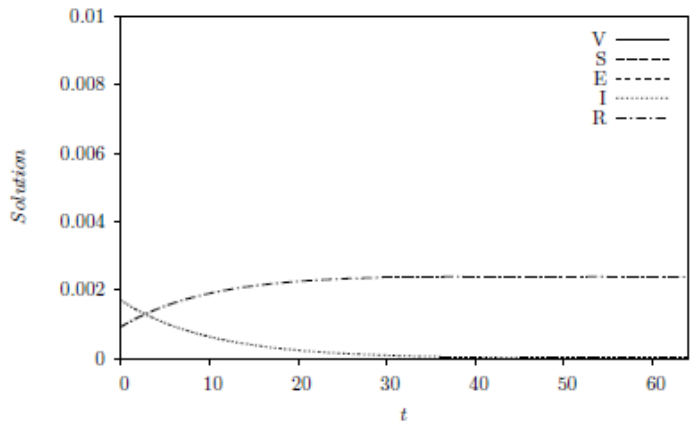

(b)

Figure 1. The number of spreading of Tuberculosis; (a) $u(t), w(t), x(t)$, (b) $y(t), z(t)$ which are obtained using RK4 for $\Delta t=0.0001$.

Table 2. The Absolute Error of $4^{\text {th }}$ Iterate SVIM Solutions compared to RK4 with $\Delta t=0.001$

\begin{tabular}{cccccc}
\hline \multirow{2}{*}{$t$} & \multicolumn{5}{c}{ SVIM } \\
\cline { 2 - 6 } & $\Delta u$ & $\Delta w$ & $\Delta x$ & $\Delta y$ & $\Delta z$ \\
\hline 5 & $6.619 \mathrm{E}-04$ & $1.190 \mathrm{E}-05$ & $9.456 \mathrm{E}-08$ & $5.908 \mathrm{E}-11$ & $6.061 \mathrm{E}-07$ \\
10 & $2.032 \mathrm{E}-03$ & $8.562 \mathrm{E}-05$ & $2.326 \mathrm{E}-07$ & $6.043 \mathrm{E}-11$ & $9.736 \mathrm{E}-07$ \\
15 & $4.102 \mathrm{E}-03$ & $2.578 \mathrm{E}-04$ & $5.142 \mathrm{E}-07$ & $2.504 \mathrm{E}-11$ & $1.195 \mathrm{E}-06$ \\
20 & $6.833 \mathrm{E}-03$ & $5.607 \mathrm{E}-04$ & $1.046 \mathrm{E}-06$ & $1.677 \mathrm{E}-11$ & $1.328 \mathrm{E}-06$ \\
25 & $1.017 \mathrm{E}-02$ & $1.021 \mathrm{E}-03$ & $2.062 \mathrm{E}-06$ & $1.235 \mathrm{E}-11$ & $1.407 \mathrm{E}-06$ \\
30 & $1.403 \mathrm{E}-02$ & $1.662 \mathrm{E}-03$ & $3.779 \mathrm{E}-06$ & $4.940 \mathrm{E}-12$ & $1.452 \mathrm{E}-06$ \\
35 & $1.836 \mathrm{E}-02$ & $2.502 \mathrm{E}-03$ & $6.441 \mathrm{E}-06$ & $3.878 \mathrm{E}-12$ & $1.477 \mathrm{E}-06$ \\
40 & $2.307 \mathrm{E}-02$ & $3.554 \mathrm{E}-03$ & $1.032 \mathrm{E}-05$ & $1.154 \mathrm{E}-11$ & $1.490 \mathrm{E}-06$ \\
45 & $2.809 \mathrm{E}-02$ & $4.83 \mathrm{E}-03$ & $1.569 \mathrm{E}-05$ & $1.627 \mathrm{E}-11$ & $1.495 \mathrm{E}-06$ \\
50 & $3.335 \mathrm{E}-02$ & $6.338 \mathrm{E}-03$ & $2.284 \mathrm{E}-05$ & $1.861 \mathrm{E}-11$ & $1.495 \mathrm{E}-06$ \\
\hline
\end{tabular}

Table 2 shows the accuracy of VIM and the maximum error of VIM solution is $\left|10^{-02}\right|$. The solutions of SVIM (4-iterates) are compared to those of RK4. The maximum error using SVIM is now decreased to $\left|10^{-06}\right|$ if it is compared to the maximum error using VIM. It also occurs in the infected case $(y(t))$, where the maximum error of VIM solution is $\left|10^{-02}\right|$, whereas the maximum error of SVIM solution has decreased i.e. $\left|10^{-11}\right|$; see Table 2. This shows that the SVIM has better accuracy. 


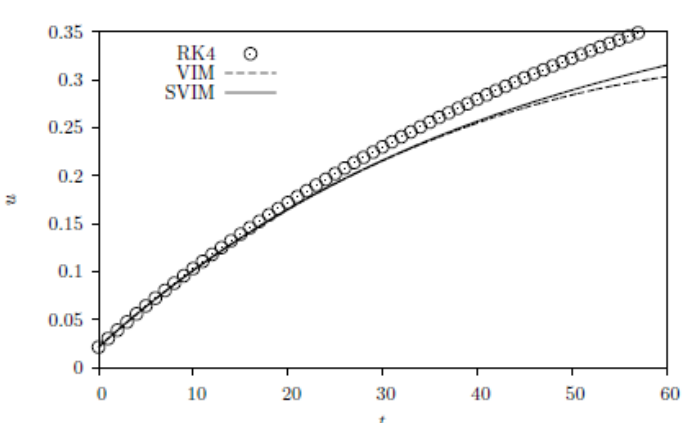

(a)

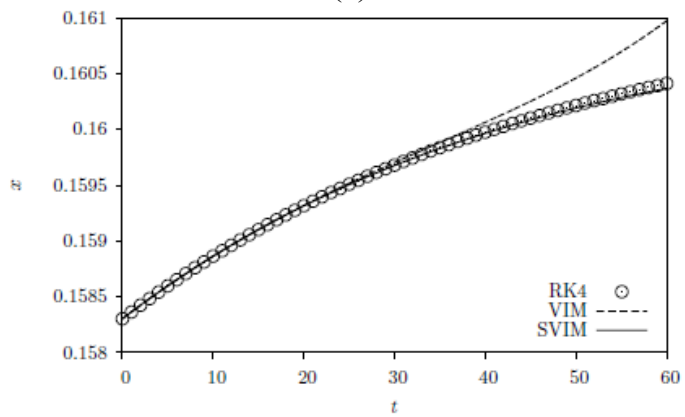

(c)

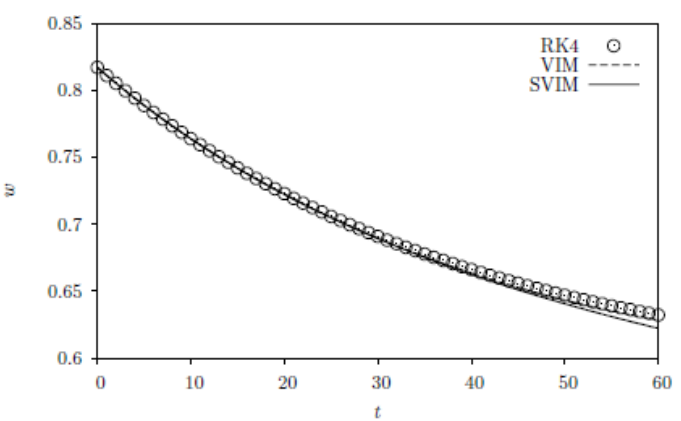

(b)

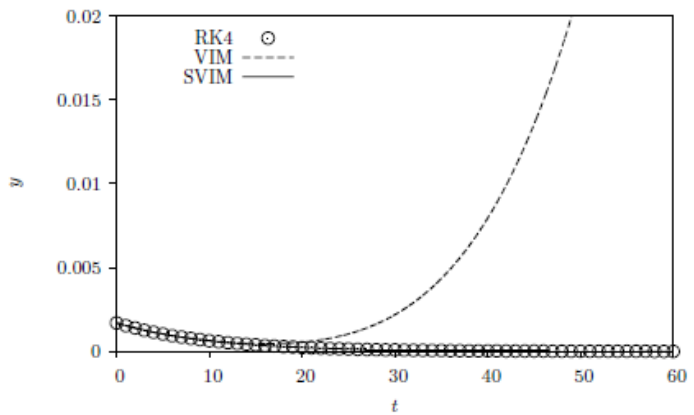

(d)

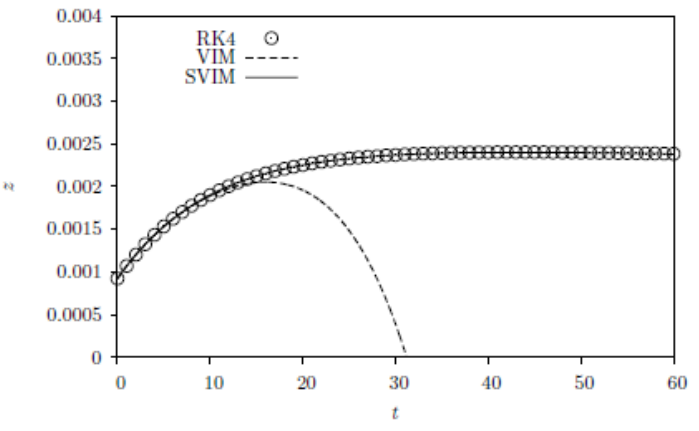

(e)

Figure 2. The approximate solution of; $(a) u(t),(b) w(t),(c) x(t),(d) y(t),(e) z(t)$ which are obtained using SVIM, VIM and RK4

Table 3. The Absolute Error of $7^{\text {th }}$ Iterate VIM Solutions compared to RK4 with $\Delta t=0.001$

\begin{tabular}{cccccc}
\hline \multirow{2}{*}{$t$} & \multicolumn{5}{c}{ VIM } \\
\cline { 2 - 6 } & $\Delta u$ & $\Delta w$ & $\Delta x$ & $\Delta y$ & $\Delta z$ \\
\hline 5 & $6.619 \mathrm{E}-04$ & $1.717 \mathrm{E}-05$ & $1.865 \mathrm{E}-09$ & $3.798 \mathrm{E}-07$ & $3.574 \mathrm{E}-07$ \\
10 & $2.033 \mathrm{E}-03$ & $9.396 \mathrm{E}-05$ & $6.975 \mathrm{E}-08$ & $1.127 \mathrm{E}-05$ & $1.061 \mathrm{E}-05$ \\
15 & $4.115 \mathrm{E}-03$ & $2.613 \mathrm{E}-04$ & $6.951 \mathrm{E}-07$ & $7.970 \mathrm{E}-05$ & $7.502 \mathrm{E}-05$ \\
20 & $6.889 \mathrm{E}-03$ & $5.376 \mathrm{E}-04$ & $3.066 \mathrm{E}-06$ & $3.139 \mathrm{E}-04$ & $2.955 \mathrm{E}-04$ \\
25 & $1.034 \mathrm{E}-02$ & $9.267 \mathrm{E}-04$ & $9.280 \mathrm{E}-06$ & $8.985 \mathrm{E}-04$ & $8.458 \mathrm{E}-04$ \\
30 & $1.445 \mathrm{E}-02$ & $1.416 \mathrm{E}-03$ & $2.245 \mathrm{E}-05$ & $2.104 \mathrm{E}-03$ & $1.980 \mathrm{E}-03$ \\
35 & $1.924 \mathrm{E}-02$ & $1.977 \mathrm{E}-03$ & $4.679 \mathrm{E}-05$ & $4.291 \mathrm{E}-03$ & $4.039 \mathrm{E}-03$ \\
40 & $2.476 \mathrm{E}-02$ & $2.564 \mathrm{E}-03$ & $8.764 \mathrm{E}-05$ & $7.916 \mathrm{E}-03$ & $7.452 \mathrm{E}-03$ \\
45 & $3.109 \mathrm{E}-02$ & $3.115 \mathrm{E}-03$ & $1.515 \mathrm{E}-04$ & $1.353 \mathrm{E}-02$ & $1.274 \mathrm{E}-02$ \\
50 & $3.834 \mathrm{E}-02$ & $3.552 \mathrm{E}-03$ & $2.459 \mathrm{E}-04$ & $2.179 \mathrm{E}-02$ & $2.051 \mathrm{E}-02$ \\
\hline
\end{tabular}




\section{CONCLUSION}

In this paper, an algorithm of VSEIR model for TB using the step variational iteration method (SVIM) was implemented. We found that SVIM is a suitable technique to solve the system of nonlinear differential equations. This method yields a solution by iterations of a rapid convergent infinite power series with enlarged intervals. Comparison between SVIM, VIM and RK4 were made; the SVIM was found to be more accurate than the VIM. SVIM is easier for calculation yet powerful and is also readily applicable to the more complex cases of problems which arise in various fields of pure and applied sciences.

\section{ACKNOWLEDGEMENT}

We gratefully acknowledge the financial support received from Direktorat Jenderal Pendidikan Tinggi (DIKTI) Indonesia no. 062/UN33.8/LL/2015.

\section{REFERENCE}

1. Judarwanto, W. (2014), Penyakit Tuberkulosis atau $T B C$, http://koranindonesiasehat.wordpress.com/2009/12 /17/penyakittuberkulosis-atau-tbc/ access date: 3th June.

2. Widaningrum, C. (2013), Pedoman Nasional pengendalian tuerculosis, Kementrian Kesehatan, Jakarta, 2014.

3. Yulita Molliq, R., Noorani, M.S.M, Ahmad, R., and Alomari, A.K., (2013), A step variational iteration method for solving non-chaotic and chaotic systems, Sains Malaysiana, 42 (3), pp. 347-358.

4. Yulita Molliq, R., Noorani, M.S.M, Ahmad, R., and Alomari, A.K., (2011), Modified step variational iteration method for solving fractional Biochemical reaction model, International Journal of Differential Equation, 2011 ID 514384.

5. Y.M. Rangkuti, Sinaga, M.S., Marpaung, F., and Side, S., (2014), A VSEIR model for transmission of tuberculosis in North Sumatera, Indonesia, AIP Conference Proceedings, 1635 (201).

6. Inokuti, M., Sekine, H., and Mura, T. (1978), General Use of the Lagrange Multiplier in Nonlinear Mathematical Physics. In: S. Nemat. Nasser, Editor, Variational Method in the Mechanics of Solids. Pergamon Press, Oxford, pp. 156- 162.

7. Alomari, A.K., Noorani. M.S.M., Nazar, R. and Li, C.P., (2010), Homotopy analysis method for solving fractional Lorenz system, Communication Nonlinear Science \& Numerical Simulation, 15 (7), pp. 1864-1872.

8. Surjantini, S.H. (2013), Profil kesehatan provinsi sumatera utara tahun 2012, Dinas Kesehatan Provinsi Sumatera utara, Medan.

9. Fredlina, K.Q. , Oka, T.B. , Dwipayana, I.M.E., (2012) Model SIR (susceptible, infectious, recovered) untuk penyebaran penyakit Tuberculosis, e-Journal Matematika, 1(1) pp. 5258. 